\title{
WEBTop: interactive 3D optics simulations on the Web
}

John Foley, Kiril Vidimce, David Banks, Taha Mzoughi

John T. Foley, Kiril Vidimce, David C. Banks, Taha Mzoughi, "WEBTop: interactive 3D optics simulations on the Web," Proc. SPIE 3831, Sixth International Conference on Education and Training in Optics and Photonics, (16 June 2000); doi: 10.1117/12.388735

Event: Education and Training in Optics and Photonics (ETOP'99), 1999, Cancun, Mexico 


\title{
WebTOP: Interactive 3D optics simulations on the Web
}

\author{
John T. Foley $^{* a}$, Kiril Vidimce ${ }^{b}$, David C. Banks $^{c}$, and Taha Mzoughi ${ }^{a}$

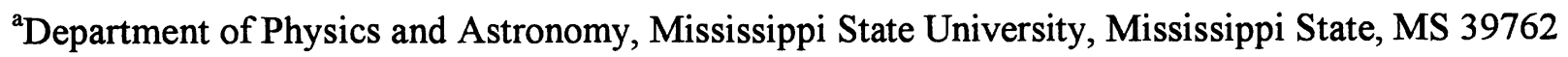 \\ bepartment of Computer Science, Mississippi State University, Mississippi State, MS 39762 \\ ${ }^{c}$ Computer Science Department, Florida State University, Tallahassee, FL 32306
}

\begin{abstract}
The Optics Project (TOP) is a 3D, interactive computer graphics system that was developed at Mississippi State University to help students learn optics. TOP runs on Silicon Graphics workstations. We describe our initial progress on WebTOP, the Web version of TOP.
\end{abstract}

Keywords: Optics, education, simulations, 3D, interactive, computer graphics, World Wide Web

\section{INTRODUCTION}

Each of us has images, vivid images, which are stored in our memories and can be recalled with a single phrase. For physicists, optical engineers, and optics students, phrases such as "the ray diagram for a thin lens" or "reflection and refraction at a planar interface" immediately bring the corresponding images to mind. These images are extremely important to us, because they often serve as the starting point when we begin to solve an optics problem or to explain an optical concept to a student.

In 1994 The Optics Project (TOP) was begun at Mississippi State University, in the Engineering Research Center for Computational Field Simulation. ${ }^{1,2}$ In a nutshell, the motivating idea behind TOP was to provide students and educators with better images to work with. In particular, TOP is a three-dimensional, interactive computer graphics system that provides the user with computer simulations of optical phenomena which are: (a) interactive, (b) three-dimensional, i.e., they allow the user to view the phenomenon of interest from any desired angle, and (c) animated (for those phenomena for which animation is appropriate). TOP has been completed, and will be discussed further in the following section.

TOP was designed to run on Silicon Graphics workstations, with the idea that upon completion it would be ported to run as an application on PCs. While we were working on TOP, it became clear that with the incredible growth of the Internet and the World Wide Web, it made more sense to port TOP to the Web than to the PC.

We have begun the port of TOP to the Web, and we call this web version WebTOP. We have ported three TOP submodules to the Web, using VRML 2.0 and Java. We will be working for the next two years under the sponsorship of the National Science Foundation, to: (a) port all the TOP modules to the Web, (b) add a Lasers module, and (c) add session logging and collaborative capabilities to WebTOP. The purpose of the present paper is to provide the reader with an introduction to WebTOP.

\section{THE OPTICS PROJECT (TOP)}

Since WebTOP is a ported version of TOP, we will begin by discussing TOP.

\footnotetext{
" Correspondence: Email: foley@erc.msstate.edu; Telephone: 601325 21918; Fax 6013258898
} 


\subsection{The TOP Modules}

TOP is comprised of seven modules. The Wave Simulation module simulates waves in a ripple tank. The user can insert several monochromatic point sources or "line" sources, and can interactively vary the properties of each source: wavelength, location (point sources), direction of propagation (line sources), etc. In the Reflection and Refraction module a monochromatic plane wave is incident upon a planar interface which separates two homogeneous media. The user controls the properties of the incident field (wavelength, angle of incidence, amplitude of the two components, phase difference between them, etc.) and the indices of refraction of the two media. The time-varying incident, reflected, and refracted electric field vectors along the corresponding ray paths are displayed on the screen.

The Geometrical Optics module simulates the behavior of light as it passes through lenses and stops on an optical "bench". The user can use either a two-dimensional object (five points sources in the shape of a $\mathrm{T}$ ) or a three-dimensional object (a large number of point sources on the surface of a tea pot). The user can put several lenses and stops on the bench; the position, diameter, and focal length of each lens can be varied interactively, as can the position and diameter of each stop. Each point source on the object emits a large number of rays at random angles, and these rays travel through the system according to the laws of geometrical optics. A movable observation screen allows the user to see the ray distribution in any plane perpendicular to the axis of the optical "bench".

In the Polarization module the propagation of a monochromatic completely polarized plane wave of light and the effect of various optical elements (polarizers and wave plates) on the corresponding electric field vector are simulated. The user controls the optical properties of the incident field, and the type, location, and characteristics of the optical elements being used. The Interference module is comprised of two separate submodules: (a) Michelson Interferometer and (b) Fabry-Perot Interferometer. In the Michelson Interferometer submodule light from a monochromatic point source illuminates the interferometer. The user controls: (a) the wavelength of the incident light, (b) the position of the translation mirror (the one that creates the path length difference), (c) the tilt of the tilt mirror, and (d) the size and position of the observation screen. In the Fabry-Perot submodule light from a monochromatic point source is incident upon a slab of material with parallel, coated faces. The user controls the wavelength of the incident light and the characteristics of the interferometer (reflectivity of the faces, distance between the faces, and the index of refraction of the slab). The interference pattern generated is displayed on an observation screen.

The Fraunhofer Diffraction module is comprised of four separate submodules: (a) Single Slit, (b) N Slit, (c) Diffraction Grating, and (d) Rayleigh Resolution Criterion. The Fresnel Diffraction module is comprised of three separate submodules: (a) Fresnel Single Slit, (b) Fresnel Double Slit, and (c) Fresnel Circular. In these modules either a monochromatic plane wave or the light from a monochromatic point source is incident upon an aperture (or set of apertures) and the corresponding intensity pattern is viewed on an observation screen. The user can vary properties of the sources or incident fields, the locations and sizes of the apertures, and the location of the observation screen.

\subsection{Use of TOP in the Classroom}

TOP has been used to help teach junior/senior level optics courses at: (a) Mississippi State University (MSU) in the Spring semesters of 1997 and 1998, (b) the University of North Carolina at Chapel Hill (UNC-CH) in the Spring semesters of 1998 and 1999, and (c) North Carolina A\&T State University (NCA\&T) in the Spring semester of 1999. It will be used to help teach the junior/senior level optics courses at all three institutions in the Spring semester of 2000.

At MSU TOP was used in three different ways. First, this class was taught in a video classroom, and TOP was used to present material to the class. The use of TOP changed the classroom dynamic in several ways. First, it allowed the instructor present the material in a much memorable way; for example, once the students have seen the electric field vector of a circularly polarized beam of light snaking its way across the computer screen, they will remember that image and be ready to work from it. In addition, TOP allows you to demonstrate difficult concepts that you cannot show otherwise, such as the phase change upon reflection of an electric field vector. Finally, and most importantly, the use of TOP led to a lot of "what happens if we do this" kinds of questions from the students, followed by the instructor entering the appropriate parameter values into TOP, then followed by a discussion.

The second way that TOP was used in this class was that the students were required to use it to solve some homework problems. When they had finished using TOP to simulate the problem in question, they then used ftp to send an image from their simulation to the professor. The third way TOP was used was in the students' class projects. The class was divided up 
into two person teams, and each team was required to use TOP to make a two to three minute video tape (Spring of 1997) or QuickTime movie (Spring of 1998) of a simulation of an optical phenomenon of their choice. On the last day of class they presented their video tape or QuickTime movie to the class and answered questions on it

At UNC-CH in the Spring of 1998 the students used four modules outside of class to do detailed assignments provided by their professor. The professor also had the students complete a very thorough evaluation form on TOP. The students responses were very positive, as had also been the case with the student evaluations done at MSU. When we talked with the professor at midterm, she felt that TOP had helped her students with concepts, in that they answered her qualitative test questions noticeably better than previous classes had. In her letter to us after the class was completed, she said "I think it was a success! " She also gave us suggestions for improving TOP. ${ }^{3}$ At UNC-CH in the Spring of 1999, a different professor taught the class, and the use of TOP was more informal, but he felt that "it was an excellent learning experience for the students" and that "the students liked exploring the things that interested them" with TOP. ${ }^{4}$

At NCA\&T in the Spring of 1999, three TOP modules and three WebTOP submodules were used by the professor in a computer lab to demonstrate concepts, and the students worked with the modules on their own outside of class. The professor felt that the use of these modules "went very well."

\subsection{TOP Web Site}

The URL for TOP's web site is http://www.erc.mstate.edu/ foley/newtop. You will find there, for each module, (a) a discussion of the theory behind each module, (b) still pictures created from that module, and (c) QuickTime movies showing that module in action. In addition, you can also find the WebTOP Fresnel Single Slit module there as well. There are approximately 70 still pictures and 40 QuickTime movies of optical phenomena at the TOP web site

\subsection{Awards and Recognitions}

Two members of our group received, jointly, a Phil Hardin Foundation Technology Award from the Mississippi Institutes for Higher Learning in 1997 for their work on TOP. An image from the TOP Web site has been included in one of the most widely used undergraduate optics text books, ${ }^{6}$ and has been chosen by Springer-Verlag to be the background picture for the cover of all the books in their Optical Sciences Series. Twelve QuickTime movies recorded from TOP sessions by an MSU undergraduate physics major were included on the "Interactive Student Tutorial," a CD-ROM that accompanies a recent general physics textbook. These movies can be found on the World Wide Web at www.mhhe.com/physsci/physical/jones/onlibr.mhtml .

\section{THE WEBTOP USER INTERFACE}

Currently WebTOP has three submodules: Fresnel Diffraction - Single Slit, Fraunhofer Diffraction - Rayleigh Resolution, and Fresnel Diffraction - Circular Aperture. The URL for WebTOP's homepage is http://www.erc.msstate.edu/ foley/webtop/ .

Let's begin our discussion of WebTOP with a tour of its interface. The WebTOP user interface for each module has five basic parts: the Modules Menu, the Scene, the Dashboard of the VRML browser, the Parameters List, and the Activities Menu (see Fig. 1).

\subsection{The Modules Menu}

The Modules Menu is located at the top of the window. It allows the user to change to a different module by simply clicking on the module's name.

\subsection{The Scene}

The Scene is the interactive 3D simulation itself. It is located directly below the Modules Menu. For example, in the Fresnel Single Slit module (see Fig. 1) the scene consists of a single slit, an observation screen, and four "widgets" which provide the user with one way to interact with the simulation by changing its parameter values. (A second way will be mentioned later.) Let us consider the Fresnel Single Slit module shown in Fig. 1. This module is simulating a monochromatic plane wave of 
light normally incident upon a single slit. The observed intensity pattern is displayed on the observation screen, and is plotted (see the white curve) above the observation screen. There are three parameters which the user can vary: (a) the wavelength of the light, (b) the width of the slit, and (c) the distance, call it $z$, from the plane containing the slit to the observation plane.

For each of these parameters, there is an object (called a widget) in the scene which the user can use to change the parameter: for the wavelength it is the white wheel in front of the slit, for the slit it is either of the two red cones located on the edges of the slit, and for $z$ it is the red cone on the top of the observation screen. These widgets are shown in Fig. 2.

Suppose the user wants to change $z$. To do this she first places the mouse cursor over the widget on the top of the observation screen. When she does this, two things happen: (a) the cursor changes appearance (it turns into a starburst), indicating that the widget has been selected, and (b) a help message appears below the dashboard of the VRML browser saying "Use these red cones to adjust the distance of the observation screen from the slit" (see Fig. 2). The user then clicks on the widget with her left mouse button and drags the observation screen to the desired position. Similar comments apply to the two other types of widgets.

\subsection{The VRML Browser Dashboard}

The VRML browser dashboard is located directly below the scene. The VRML browser is a plug-in to Netscape or Microsoft Internet Explorer and it has to be installed before you can use the modules. We provide links to a VRML browser download from the WebTOP site. The VRML browser shown in Figs 1 and 2 is CosmoPlayer. The controls on the dashboard allow the user to use the mouse cursor to manipulate the scene in several ways. The default mode of operation for the mouse cursor is the Rotate mode. The user can rotate the scene in any desired direction in 3D space by grabbing the scene with his left mouse button and pushing or pulling the cursor in the desired direction. A second mode of operation is the Pan mode. By clicking on the button just to the right of the center button on the dashboard, the user changes the cursor from whatever mode it is in to the Pan mode. The user can now use the left mouse button to grab the scene and translate it in any direction in 3D space.

The third mode of operation is the Zoom mode. By clicking on the button just to the left of the center button on the dashboard, the mode of the cursor changes to the Zoom mode. The user can then click on his left mouse button and push towards the scene to zoom in, or pull the away from the scene to zoom out. The fourth mode of operation is the Seek mode. By clicking on the second button to the left of the center button on the dashboard, the mode of the cursor changes to the Seek mode. In this mode, when the user clicks on a location inside the scene, the viewer "seeks" it out by zooming in directly towards that point. Finally, there is an Undo Move button on the dashboard which undoes the last operation performed, and a Home button which returns the Scene to its default position.

\subsection{The Parameter List}

The Parameter List is at the bottom of the window (see Fig. 1). The purpose of the parameter list is to inform the user of what the current parameter values are and to provide her with another method (via Keyboard Entry Boxes) of changing the ones she has control over. For example, in Fig. 1, the first three items in the list are Keyboard Entry Boxes for, respectively, the wavelength of the light, the width of the slit, and the distance from the aperture plane to the observation screen. If the user uses a widget, the new value of the parameter appears in the corresponding box. Alternatively, she can type the parameter value in the box, if she so desires. The fourth entry in the list is the Fresnel number for the current situation. The Fresnel number depends on all three parameters, and its value is updated whenever the value of any one of the other three is changed. The user does not have direct control over it.

\subsection{The Activities Menu}

The Activities Menu is in the upper left-hand corner of the screen. It lists the five activities available in WebTOP. These will be discussed in the next section. 


\section{WEBTOP ACTIVITIES}

Let us now take a look at the activities available for each module.

\subsection{Module}

This is just the simulation itself, as pictured in Fig. 1. As an example of the kinds of things that can be done, consider Fig. 3. This is a set of four images from the Rayleigh Resolution submodule of the Fraunhofer diffraction module. In each figure, monochromatic light from two distant point sources is incident upon a lens. Each of the two gray bars represents the chief ray from its respective source. The light passes through the lens and is imaged on the observation screen, which lies in the back focal plane of the lens. The two images are not point images due to the diffraction which takes place when the light passes through the lens; they are circular diffraction patterns. In this submodule the user can vary: (a) the angle between the two sources, (b) the wavelength of the light, (c) the diameter of the lens and (d) the focal length of the lens.

Let $\lambda$ be the wavelength of the light, $D$ be the diameter of the lens, and $\theta$ be the angular separation of the sources, i.e., the angle between the two gray bars. According to the Rayleigh resolution criterion, the two images are resolved if and only if $\theta$ is greater than or equal to

$$
\theta_{\min }=\frac{1.22 \lambda}{D}
$$

In Fig. 3 (a), $\theta$ is twice as big as $\theta_{\min }$ and the stars are resolved. In Fig. 3 (b), $\theta$ has been decreased until it is equal to $\theta_{\min }$, and the two stars are barely resolved. In Fig. 3 (c), $\theta$ is half as big as $\theta_{\min }$ and the stars are not resolved. In Fig. 3(d), the $\theta$ and the diameter of the lens are the same as they were in Fig. 3(b), but the wavelength has been decreased from $550 \mathrm{~nm}$ to $450 \mathrm{~nm}$. Upon comparing (d) to (b), we see that in the latter the resolution is better, because $\theta_{\min }$ is larger.

\subsection{Controls}

When the user clicks on Controls in the activities menu, she is linked to an HTML document which explains (a) how to manipulate the scene (i.e., how to use Rotate, Pan, Zoom, etc.) and (b) how to change the parameter values (i.e., how to use the widgets and parameter entry boxes).

\subsection{Theory}

When the user clicks on Theory in the activities menu, she is linked to an HTML document which contains the theory relevant to the current module. These are the relevant sections of lecture notes of the junior/senior undergraduate optics course taught at Mississippi State University. For example, in the Fresnel Diffraction, Circular Aperture submodule the theory section has sections on the relevant diffraction integral, the on-axis intensity and the Fresnel number, Fresnel zones, and the full intensity pattern. It is suggested that the user read this material before interacting with the module, but this is not mandatory.

\subsection{Examples}

When the user clicks on Examples in the Activities menu, she is liked to an HTML page that offers the opportunity to view any of several previously recorded WebTOP sessions. When the user clicks on one of the choices, the session is replayed in the Scene part of the window. These example sessions are very helpful to a new user because it gives her a feel for what can be done with the module.

It is important to note that these previously recorded sessions are not just "movies." They are fully 3D in the sense that the user can use all the VRML browser commands (Rotate, Pan, Zoom, etc.) to change the way the scene is being viewed. The user can even change the parameters while the example is running.

\subsection{Exercises}


When the user clicks on Exercises in the Activities module, she is linked to an HTML page with a list of exercises she can try. These are inquiry-based exercises in that the user is asked to interact with the software, observe how the simulation changes, and then come up with an explanation for what is happening. The following is one of the exercises from the Fresnel Diffraction Circular Aperture submodule.

Exercise 6: Changing $\mathrm{z}$, the distance from the aperture plane to the observation plane: odd Fresnel numbers.

Set the wavelength of the light to be $500 \mathrm{~nm}$ and the diameter of the aperture to be $1.0 \mathrm{~mm}$.

(a) Use the red cone widget on the top of the observation screen to make the wavelength of the light such that the Fresnel number is 5.0 . Is the intensity on axis a maximum or a minimum? Starting at one edge of the intensity pattern and proceeding across it through the origin to the other edge, how many "major" maxima do you encounter?

(b) Use the red cone widget on the top of the observation screen to make the wavelength of the light such that the Fresnel number is 7.0 . Is the intensity on axis a maximum or a minimum? Starting at one edge of the intensity pattern and proceeding across it through the origin to the other edge, how many "major" maxima do you encounter?

(c) Use the red cone widget on the top of the observation screen to make the wavelength of the light such that the Fresnel number is 9.0 . Is the intensity on axis a maximum or a minimum? Starting at one edge of the intensity pattern and proceeding across it through the origin to the other edge, how many "major" maxima do you encounter?

(d) What rule of thumb can be deduced from the results seen in (a) through (c) above, as regards the intensity on axis when the Fresnel number is odd? Use Fresnel zones to explain why this is happening.

(e) What rule of thumb can be deduced from the results seen in (a) through (c) above, as regards the number of major maxima seen across the intensity pattern when the Fresnel number is odd? Use Fresnel zones to explain why this is happening.

Figs. 4(a) and (b) show, respectively, the diffraction patterns when the Fresnel numbers are five and four.

\section{CONCLUSION}

As each module is ported from TOP to the Web, it will be added to WebTOP at the URL given in Section 3. We encourage you to use the modules, and to give us feedback in the form of questions or comments. We can be contacted by email at foley@erc.msstate.edu or at top@cs.msstate.edu. If you are planning to use WebTOP to help teach an optics course, please feel free to contact us for information about how others have used it to help teach their classes. In the summer of 2000 we will hold a WebTOP workshop at Mississippi State University. Please contact us if you are interested in attending it.

\section{ACKNOWLEDGEMENTS}

We would like to acknowledge support for TOP from the National Science Foundation under grant DUE 9555053 and for WebTOP under grant DUE 9950569.

\section{REFERENCES}

1. D. C. Banks, J. M. Brown, J. T. Foley, K. N. Vidimce, and M. Kiu, "Interactive 3D visualization of optical phenomena," IEEE Computer Graphics and Applications 18, pp. 66-69, 1998.

2. J. T. Foley and D. C. Banks, "The Optics Project," Optics and Photonics News 9, pp.45 and 50, 1998.

3. L. E. MacNeill, private communication. 
4. J. Glessner, private communication.

5. F. James, private communication.

6. E. Hecht, Optics, third ed., Addison-Wesley, Reading MA, 1998.

7. E. Jones and R. Childers, Contemporary College Physics 3/e, McGraw-Hill, New York, 1998. 


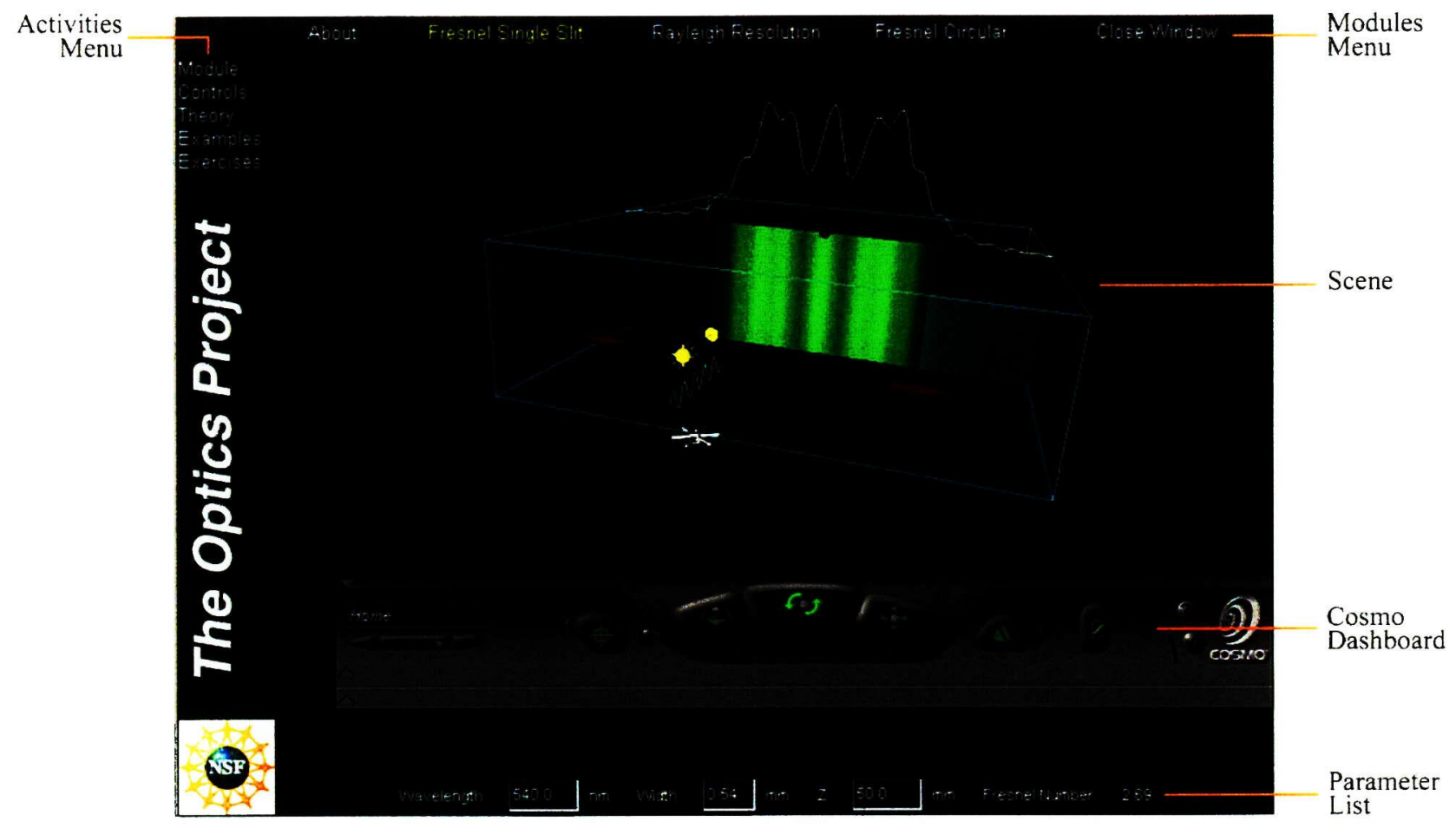

Fig.1 Typical WebTOP window

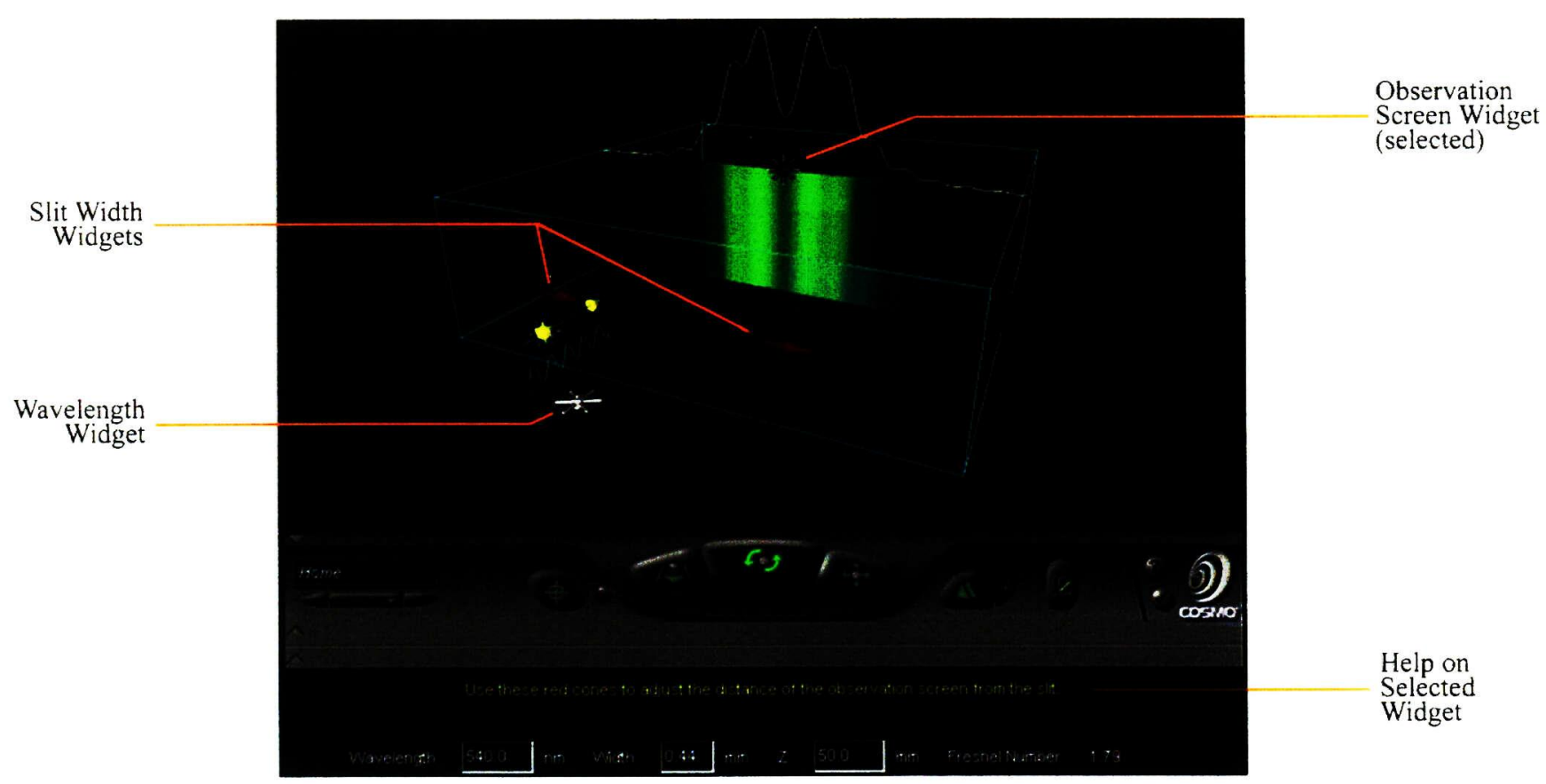

Fig.2 3D widgets and widget help. 
(a)

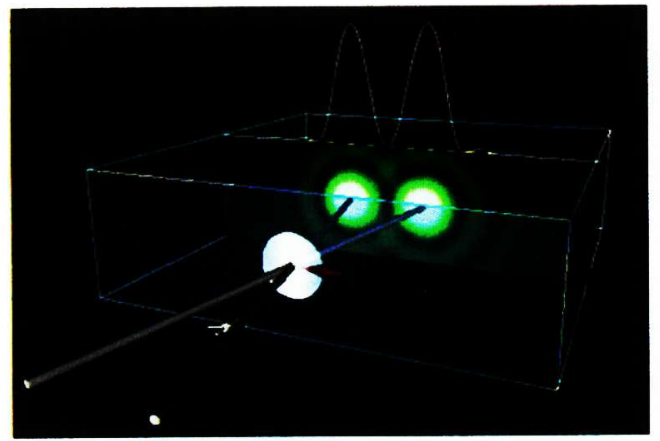

(c)

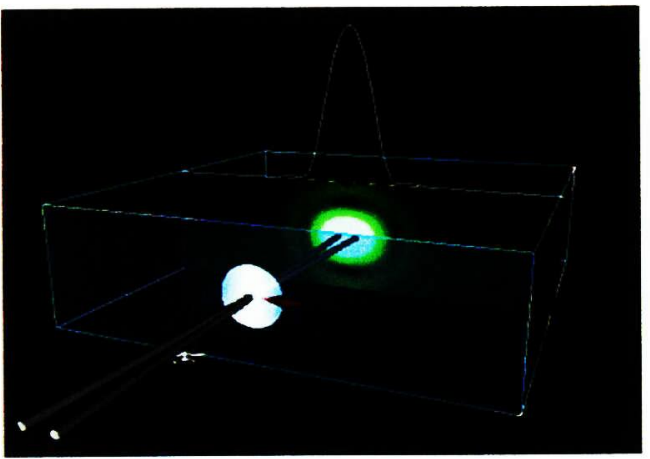

(b)

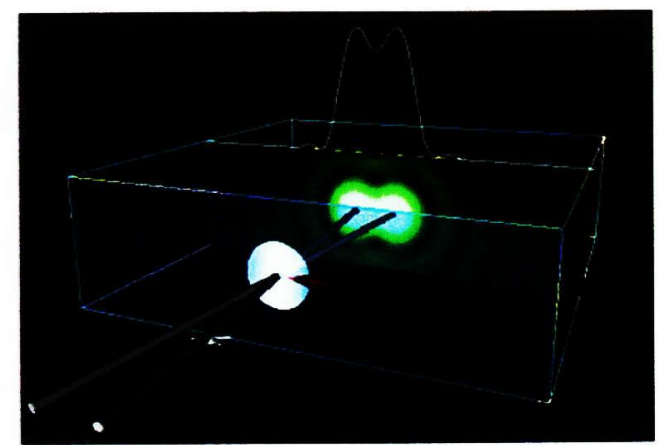

(d)

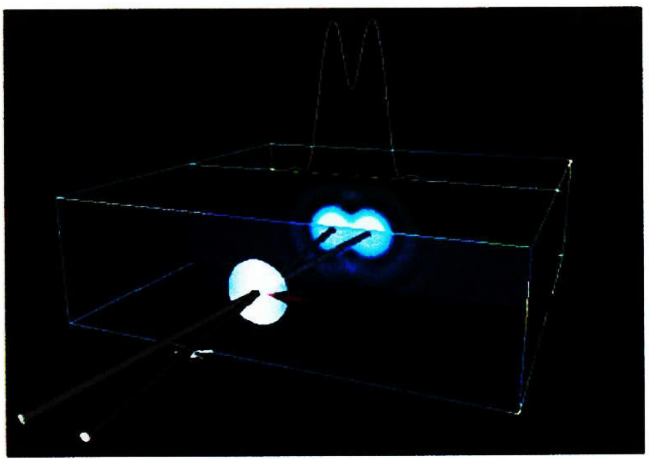

Fig.3 Rayleigh Resolution Module.

(a) wavelength $=550 \mathrm{~nm}$, diameter $=3.355 \mathrm{~mm}$, angle $=4.0 \times 10^{-4}$ rads. The images are resolved.

(b) wavelength $=550 \mathrm{~nm}$, diameter $=3.355 \mathrm{~mm}$, angle $=2.0 \times 10^{-4}$ rads. The images are barely resolved.

(c) wavelength $=550 \mathrm{~nm}$, diameter $=3.355 \mathrm{~mm}$, angle $=1.0 \times 10^{-4}$ rads. The images are not resolved.

(d) wavelength $=450 \mathrm{~nm}$, diameter $=3.355 \mathrm{~mm}$, angle $=2.0 \times 10^{-4}$ rads. The images are resolved.

(a)

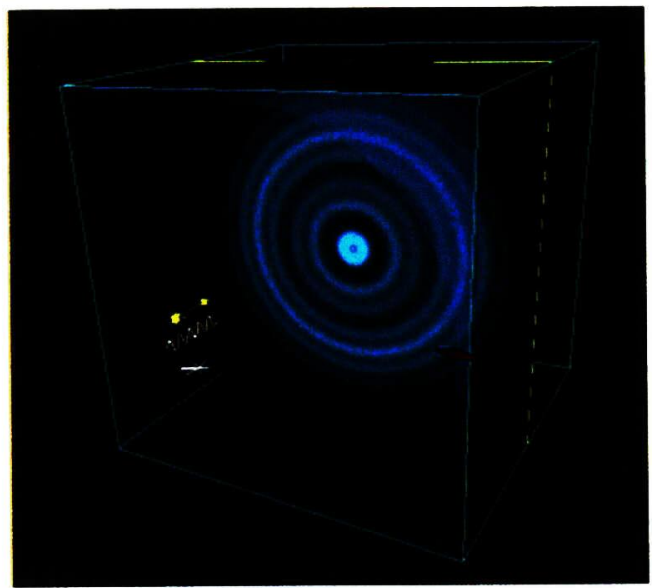

(b)

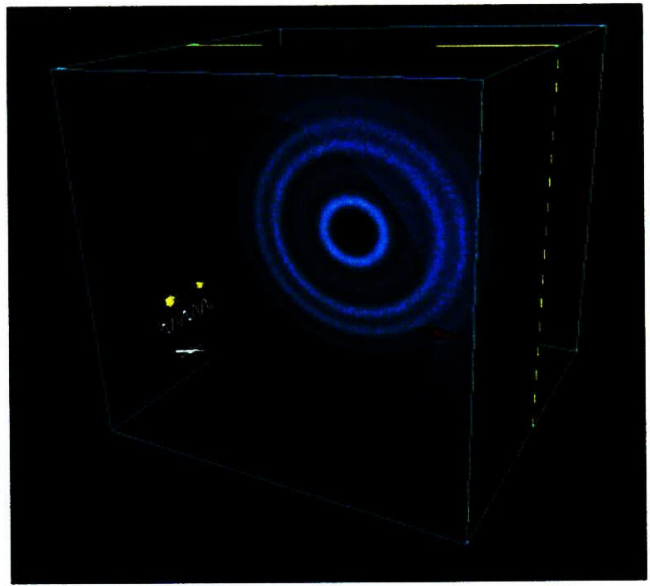

Fig.4 Fresnel Diffraction, Circular Aperture. (a) Fresnel number $=5$. (b) Fresnel number $=4$. 